\title{
A Gaussian Process Regression Approach to Model Aircraft Engine Fuel Flow Rate
}

\author{
Yashovardhan S. Chati* \\ Massachusetts Institute of Technology \\ 77 Massachusetts Avenue \\ Cambridge, Massachusetts 02139 \\ yschati@mit.edu
}

\author{
Hamsa Balakrishnan \\ Massachusetts Institute of Technology \\ 77 Massachusetts Avenue \\ Cambridge, Massachusetts 02139 \\ hamsa@mit.edu
}

\begin{abstract}
The problem of building statistical models of cyber-physical systems using operational data is addressed in this paper, using the case study of aircraft engines. These models serve as a complement to physics-based models, which may not accurately reflect the operational performance of systems. The accurate modeling of fuel flow rate is an essential aspect of analyzing aircraft engine performance. In this paper, operational data from Flight Data Recorders are used to model the fuel flow rate. The independent variables are restricted to those which are obtainable from trajectory data. Treating the engine as a statistical system, an algorithm based on Gaussian Process Regression (GPR) is developed to estimate the fuel flow rate during the airborne phases of flight. The algorithm propagates the uncertainty in the estimates in order to determine prediction intervals. The proposed GPR models are evaluated for their predictive performance on an independent set of flights. The resulting estimates are also compared with those given by the Base of Aircraft Data (BADA) model, which is widely used in aircraft performance studies. The GPR models are shown to perform statistically significantly better than the BADA model. The GPR models also provide interval estimates for the fuel flow rate which reflect the variability seen in the data, presenting a promising approach for data-driven modeling of cyber-physical systems.
\end{abstract}

\section{CCS CONCEPTS}

-Computing methodologies $\rightarrow$ Modeling methodologies; Modeling and simulation; Model development and analysis; • Applied computing $\rightarrow$ Aerospace; Physical sciences and engineering;

\section{KEYWORDS}

Aircraft engine fuel flow rate, Flight Data Recorder, Statistical analysis, Gaussian Process Regression, Base of Aircraft Data (BADA)

ACM Reference format:

Yashovardhan S. Chati and Hamsa Balakrishnan. 2017. A Gaussian Process Regression Approach to Model Aircraft Engine Fuel Flow Rate. In

${ }^{*}$ Corresponding author (ORCID: 0000-0001-7664-4230)

Permission to make digital or hard copies of all or part of this work for personal or classroom use is granted without fee provided that copies are not made or distributed for profit or commercial advantage and that copies bear this notice and the full citation on the first page. Copyrights for components of this work owned by others than ACM must be honored. Abstracting with credit is permitted. To copy otherwise, or republish, to post on servers or to redistribute to lists, requires prior specific permission and/or a fee. Request permissions from permissions@acm.org.

ICCPS 2017, Pittsburgh, PA USA

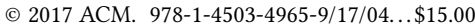

DOI: http://dx.doi.org/10.1145/3055004.3055025
Proceedings of The 8th ACM/IEEE International Conference on Cyber-Physical Systems, Pittsburgh, PA USA, April 2017 (ICCPS 2017), 10 pages. DOI: http://dx.doi.org/10.1145/3055004.3055025

\section{INTRODUCTION}

The accurate modeling of a cyber-physical system (CPS) is essential for evaluating its performance, and for the development of control algorithms. These models have traditionally been purely physicsbased, and have relied on a detailed knowledge of the underlying designs, processes, and parameters. Typical physics-based models simulate system behavior by solving a set of differential equations governing the system dynamics. They are useful to identify the important factors influencing system behavior, and to give an idealized representation of system performance. However, a disadvantage of such models is that they may not accurately reflect the operational performance of the system in real-world environments, due to simplifying assumptions that neglect uncertainties, as well as unmodeled effects. Moreover, such models often contain parameters which are difficult to estimate. With the increase in sensor and computing technologies, statistical models based on data provide a promising way to analyze system behavior, especially when they effectively leverage the advantages of physics-based models (for example, to guide variable selection).

An aircraft and its engine form a complex CPS, with numerous sensors that monitor various aircraft and engine parameters during flight. The control of the engine is achieved automatically through a digital controller in the engine. Combustion of fuel inside the engine provides the energy to propel the aircraft. Therefore, the fuel flow rate (mass of fuel injected into the engine per unit time) is one of the primary variables governing aircraft and engine operations. Modeling of the fuel flow rate is needed to evaluate other performance metrics of the aircraft engine, to assess airline costs (fuel constitutes a major component of the direct operating costs), and to gauge the environmental impacts of aviation (fuel burn results in the generation of pollutants).

Fuel flow rate modeling has received attention in prior literature (for example, [24, 27]). Most of these studies have used data from simulation software, flight manuals, ground tests, or performance calculators. Being non-operational in nature, such data may not be reflective of the performance of a real engine in flight. Indeed, statistically significant differences have been shown to exist between the fuel burn estimates from ground test-based methods (for example, the International Civil Aviation Organization Aircraft Engine Emissions Databank [12]) and the actual values seen in flight operations $[6,17]$. Therefore, the use of operational flight data to develop models of fuel flow rate is expected to give estimates which better 
reflect the behavior of an aircraft in flight. Prior studies which have used operational data, although limited in number, have illustrated the benefits of using such data $[13,22,25,26,30,31]$.

\subsection{Contributions of this Paper}

In this paper, we use operational data from Flight Data Recorders (FDRs) to develop models of aircraft engine fuel flow rate. The FDR is a high-fidelity source of data since it records actual flight parameters (as a function of time) on board the aircraft. However, these archives are generally proprietary to airlines and are not easily accessible. By contrast, flight trajectories can be obtained from surveillance systems. The development of fuel burn and emissions inventories require the ability to translate individual aircraft trajectories to estimates of fuel burn, without access to FDR data from those particular flights. We would therefore, like to develop statistical, data-driven models that can translate aircraft trajectory variables into estimates of fuel flow rate. We model the aircraft engine via a statistical approach, instead of the more common deterministic approach, to account for various random disturbances internal and external to the engine [14]. Machine learning algorithms then provide a powerful tool to model the system using data, especially when the modeling objective is the ability to make predictions on new data. In this paper, we use Gaussian Process Regression (GPR) to model the fuel flow rate, in order to obtain estimates of fuel flow rate and the associated confidence intervals. A novel algorithm is developed which uses GPR to propagate the takeoff aircraft mass and fuel flow rate (initial conditions) to estimate the fuel flow rate as a function of time. The use of a statistical modeling technique enables quantifying the uncertainty in fuel flow rate at each time instant. The algorithm takes care to propagate this uncertainty in fuel flow rate from one time instant to the next. The resulting GPR models are found to give mean errors of $2.9 \%, 6.2 \%$, and $15.3 \%$ in ascent, cruise, and descent, respectively. They are also shown to perform statistically significantly better than the current state-ofthe-art aircraft performance models with up to $50 \%$ reduction in mean error.

We believe the modeling methodology proposed in this paper can find general applicability across different CPS domains. In general, the paper demonstrates how to:

(1) develop a statistical framework to build models which can propagate initial conditions to estimate system performance, along with its associated uncertainty, at each time instant in the future,

(2) use a physical understanding of system dynamics to yield the input features suitable for building the statistical model,

(3) handle input variables which need to be estimated in tandem with the output variable,

(4) handle input variables which are unknown and have uncertainty associated with them, and

(5) properly propagate uncertainty in the unknown input and output variables from one time instant to the next.

\subsection{Outline}

We start with a brief description of the data set in Sec. 2. We describe the choice of the modeling variables in Sec. 3. This is followed by a brief primer on Gaussian Process Regression in Sec.
4. We explain our algorithm for fuel flow rate modeling in Sec. 5. In Sec. 6, we evaluate the predictive performance of our algorithm on independent test data. We compare our model results with those given by the Base of Aircraft Data (BADA) model in Sec. 7. The main conclusions of the paper and some directions for future work are highlighted in Sec. 8.

\section{DATA SET}

The FDR data used in this study come from the Airbus A321-100 aircraft of a major European carrier. The A321-100 is driven by two CFMI CFM56-5B1/2 or 2P turbofan engines. There are 117 flights of the A321-100 in our data set, with 783 - 1436 data points per flight. Each point corresponds to one observation in the FDR data set. The FDR data set reports parameters such as the aircraft trajectory, speeds, gross mass, acceleration, fuel flow rate, engine temperatures, ambient pressure and temperature, positions of auxiliary devices and control surfaces, etc. as a function of time in flight. Before analysis, each flight profile is divided into different phases using criteria solely based on the aircraft trajectory, speeds, and acceleration values [6]. In this paper, we focus on the airborne phases of flight, namely, ascent (just after takeoff from the departure airport to top of climb where cruise starts), cruise (marked by long periods of level altitude), and descent (from end of cruise to just before touchdown at the arrival airport). The frequency of observations varies by phase, being more frequent in the rapidly changing phases of flight (such as near takeoff and touchdown, where data are collected at $1 \mathrm{~s}$ intervals), and less frequent in the more constant phases of flight (such as cruise, where data are collected at $150 \mathrm{~s}$ intervals).

\section{MODELING VARIABLES}

All the modeling variables are metric and continuous in nature. Hence, the fuel flow rate estimation problem is fundamentally a regression problem. The choice of the variables for regression is made from an understanding of the physics governing aircraft operations. As mentioned in Sec. 1, we would like our models to not require access to additional FDR data. We therefore, restrict our input variables to those which are available from ground-based surveillance systems.

For an aircraft in flight, the equations of motion can be written, in a simplified form, as follows:

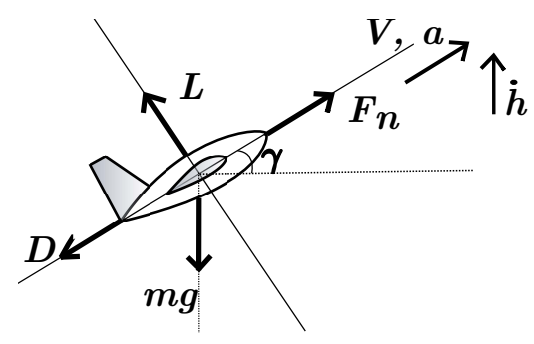

Figure 1: Forces on an aircraft in flight (simplified). 


$$
\begin{aligned}
L & =m g \cos \gamma \\
F_{n} & =D+m g \sin \gamma+m a \\
L & =q \mathcal{S} C_{L} \\
\sin \gamma & =\frac{\dot{h}}{V} \\
D & =q \mathcal{S}\left(C_{D_{0}}+C_{D_{2}} C_{L}^{2}\right) \\
q & =\frac{1}{2} \rho V^{2}
\end{aligned}
$$

Here, $L$ is the lift on the aircraft, $m$ is the aircraft gross mass, $g$ is the acceleration due to gravity, $\gamma$ is the flight path angle, $F_{n}$ is the aircraft net thrust from all the engines, $D$ is the aircraft drag, $a$ is the aircraft acceleration in the thrust direction, $q$ is the dynamic pressure, $\mathcal{S}$ is the reference wing area, $C_{L}$ is the aircraft lift coefficient, $\dot{h}$ is the vertical speed, $V$ is the true air speed, $C_{D_{0}}$ and $C_{D_{2}}$ are aircraft drag coefficients, and $\rho$ is the ambient air density. The aircraft angle of attack and the bank angle are neglected, since these quantities cannot be obtained from flight track data. Combining Eqs. 1-6, we have

$$
F_{n}=q \mathcal{S} C_{D_{0}}+\frac{C_{D_{2}} m^{2} g^{2}}{q \mathcal{S}}-\frac{C_{D_{2}} m^{2} g^{2} \dot{h}^{2}}{q \mathcal{S} V^{2}}+m g \frac{\dot{h}}{V}+m a .
$$

The fuel flow rate is related to the net thrust via the Thrust Specific Fuel Consumption (TSFC):

$$
\dot{m}_{f}=\frac{\text { TSFC } \times F_{n}}{N_{\text {eng }}}
$$

Here, $\dot{m}_{f}$ is the fuel flow rate per engine (averaged over all engines), and $N_{\mathrm{eng}}$ is the number of engines (2 for the A321-100). Therefore, from Eq. 7 and Eq. 8, in the airborne phases of flight, the approximate functional relation for the fuel flow rate can be written as

$$
\dot{m}_{f} \approx \dot{m}_{f}\left(q \mathcal{S}, m, \frac{\dot{h}}{V}, a, C_{D_{0}}, C_{D_{2}}, \mathrm{TSFC}\right) .
$$

We make further simplifying assumptions in order to restrict ourselves to variables which are available through ground-based tracking data.

- When the true ambient density (required for the calculation of the dynamic pressure q) is not available, we assume a surrogate density value according to the International Standard Atmosphere (ISA) model [2], which models the density as a function of the altitude (available from groundbased tracking systems). The equations for the density variation in the ISA model are given in Appendix A.

- Aircraft performance depends on the true air speed $V$, which is the aircraft velocity with respect to the surrounding air. This velocity cannot be measured on the ground. Hence, we use the ground speed $V_{\mathrm{GS}}$, which can be measured from the ground, as a surrogate for the true air speed. The ground speed is the aircraft velocity with respect to the ground.

- It is not possible to measure the aircraft acceleration (as required in Eq. 2) from the ground. We therefore, use the numerical derivative of the ground speed as a surrogate value for the aircraft acceleration, $a_{\mathrm{surr}}=\frac{\Delta\left(V_{\mathrm{GS}}\right)}{\Delta t}$, where $t$ is the time in flight. The values of the surrogate acceleration are smoothed through a low pass filter before using them for analysis to remove noise arising from numerical differentiation.

- The Base of Aircraft Data (BADA) methodology [15] is used for modeling the drag coefficients and the TSFC. According to the BADA methodology, the drag coefficients are assumed to be aircraft type specific constants in the ascent and cruise phases. However, in the descent phase, they are assumed to have discrete levels depending on the altitude of the aircraft with respect to the mean sea level elevation of the arrival airport, the aircraft speed, and the aircraft gross mass. The BADA methodology also models the TSFC to be just a function of the aircraft true air speed.

Based on the assumptions above, the functional dependence of the fuel flow rate can be approximately written as

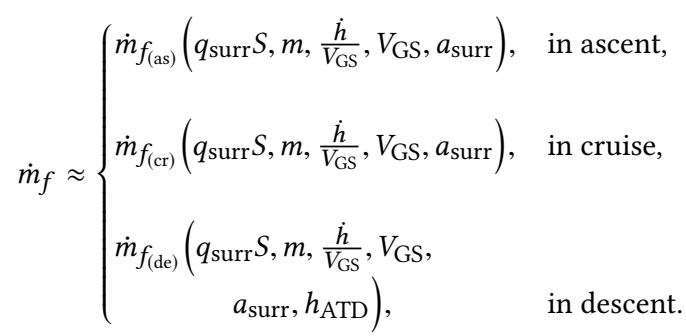

Here, the subscripts (as), (cr), and (de) refer to ascent, cruise, and descent, respectively. $q_{\text {surr }}=\frac{1}{2} \rho V_{\mathrm{GS}}^{2}$ is the surrogate dynamic pressure based on the ground speed (with density $\rho$ given by the ISA model if needed), and $h_{\text {ATD }}$ is the aircraft altitude above the mean sea level elevation of the arrival airport. Thus, in the regression models to be built, the input/independent/predictor variables are the surrogate dynamic pressure multiplied by the reference wing area $\left(q_{\text {surr }} S\right.$, in units of newton), aircraft gross mass ( $m$, in units of kilogram), the ratio of the aircraft vertical speed to its ground speed ( $\frac{\dot{h}}{V_{\mathrm{GS}}}$, a dimensionless quantity), the aircraft ground speed ( $V_{\mathrm{GS}}$, in units of meter per second), the aircraft surrogate acceleration ( $a_{\text {surr }}$, in units of meter per second per second) in ascent, cruise, and descent. In descent alone, there is an additional input variable, namely the aircraft altitude above the mean sea level elevation of the arrival airport ( $h_{\mathrm{ATD}}$, in units of meter). In all the phases, the output/dependent/predicted variable is the average fuel flow rate per engine $\left(\dot{m}_{f}\right.$, in units of kilogram per second). It should be noted that except for the aircraft gross mass, all other input variables can be obtained from easily accessible ground-based flight tracking systems. The predictor variables chosen are the primary factors affecting the fuel flow rate. However, many other secondary factors can also affect the fuel flow rate (for example, aging, component deterioration, engine bleed, etc.). These secondary factors are difficult to model. However, it is assumed that the statistically-derived models can incorporate the effects of unmodeled and neglected factors through the generation of prediction intervals (instead of just point predictions).

\section{GAUSSIAN PROCESS REGRESSION}

In this section, we briefly describe the Gaussian Process Regression (GPR) methodology. GPR is a powerful nonparametric Bayesian 
approach with a Gaussian probabilistic framework. It has found application in diverse areas, including biomedical applications and health care $[7,8,18]$, remote sensing $[11,16]$, music [19], robotics [5], cellular communications [23], and material microstructure analysis [3]. More details of this method can be found in [21, 29].

A regression model is given as

$$
y=f(\mathbf{x})+\epsilon
$$

where, $y$ is the output/dependent variable, $\mathbf{x}$ is the input vector, $f(\mathbf{x})$ is the underlying regression function that we wish to estimate, and $\epsilon$ is the noise with which the dependent variable is distributed about the regression function. Under GPR, we assume the regression function to follow a Gaussian Process (GP) prior. A function $f(\mathbf{x})$ is said to follow a Gaussian Process if the function values at any finite set of inputs $\mathbf{x}$ follow a joint Gaussian distribution [21].

$$
f(\mathbf{x}) \sim G P\left(m_{e}(\mathbf{x}), k\left(\mathbf{x}, \mathbf{x}^{\prime}\right)\right)
$$

where, $m_{e}(\mathbf{x})$ is the mean function and $k\left(\mathbf{x}, \mathbf{x}^{\prime}\right)$ is the kernel/ covariance function over two inputs $\mathbf{x}$ and $\mathbf{x}^{\prime}$, which governs the covariance among function values. Under GPR, the mean function is often assumed to be the zero function. It is common to assume the noise to follow a Gaussian distribution, $\epsilon \sim \mathcal{N}\left(0, \sigma_{n}^{2}\right)$, with mean 0 and noise variance $\sigma_{n}^{2}$. Under the assumption of a zero mean function for the GP governing the regression function and Gaussian noise, the dependent variable $y$ also follows a GP with a zero mean function and a 'noisy' kernel function $k_{\text {noise }}\left(\mathbf{x}_{\mathbf{p}}, \mathbf{x}_{\mathbf{q}}\right)$ over $d$-dimensional input vectors $\mathbf{x}_{\mathbf{p}}$ and $\mathbf{x}_{\mathbf{q}}$.

$$
y \sim G P\left(0, k_{\text {noise }}\left(\mathbf{x}_{\mathbf{p}}, \mathbf{x}_{\mathbf{q}}\right)\right)
$$

The noisy kernel function over the dependent variables $k_{\text {noise }}\left(\mathbf{x}_{\mathbf{p}}, \mathbf{x}_{\mathbf{q}}\right)$ relates to the kernel function over the regression function values $k\left(\mathbf{x}_{\mathbf{p}}, \mathbf{x}_{\mathbf{q}}\right)$ as

$$
k_{\text {noise }}\left(\mathbf{x}_{\mathbf{p}}, \mathbf{x}_{\mathbf{q}}\right)=k\left(\mathbf{x}_{\mathbf{p}}, \mathbf{x}_{\mathbf{q}}\right)+\sigma_{n}^{2} \delta_{p q}
$$

where, $\delta$ is the Kronecker delta.

The GP literature is filled with different kernel functions which affect the nature of the regression functions used for modeling. These functions give GPR great modeling flexibility. Two commonly used kernel functions are described as follows:

- Dot Product Squared Exponential (DPSE) kernel: This kernel function is used to model very smooth functions. It is given by

$$
\begin{aligned}
k\left(\mathbf{x}_{\mathbf{p}}, \mathbf{x}_{\mathbf{q}}\right)= & \sigma_{0}^{2}+\mathbf{x}_{\mathbf{p}}^{T} \Sigma \mathbf{x}_{\mathbf{q}} \\
& +\sigma_{f}^{2} \exp \left(-\frac{1}{2} \sum_{i=1}^{d} \frac{\left(x_{p, i}-x_{q, i}\right)^{2}}{\ell_{i}^{2}}\right), \\
\Sigma= & \operatorname{diag}\left(\sigma_{1}^{2}, \sigma_{2}^{2}, \ldots, \sigma_{d}^{2}\right) .
\end{aligned}
$$

- Dot Product Exponential (DPE) kernel: This kernel function is used to model very rough functions. It is given by

$$
\begin{aligned}
k\left(\mathbf{x}_{\mathbf{p}}, \mathbf{x}_{\mathbf{q}}\right)= & \sigma_{0}^{2}+\mathbf{x}_{\mathbf{p}}{ }^{T} \Sigma \mathbf{x}_{\mathbf{q}} \\
& +\sigma_{f}^{2} \exp \left(-\sqrt{\sum_{i=1}^{d} \frac{\left(x_{p, i}-x_{q, i}\right)^{2}}{\ell_{i}^{2}}}\right), \\
\Sigma= & \operatorname{diag}\left(\sigma_{1}^{2}, \sigma_{2}^{2}, \ldots, \sigma_{d}^{2}\right) .
\end{aligned}
$$

In Eqs. 15 and $16, \mathbf{x}_{\mathbf{p}}$ and $\mathbf{x}_{\mathbf{q}}$ are $d$-dimensional input column vectors, $\sigma_{0}^{2}$ is the constant variance parameter, $\sigma_{1}^{2}, \sigma_{2}^{2}, \ldots, \sigma_{d}^{2}$ are the variance parameters for each of the $d$ input dimensions, $\sigma_{f}^{2}$ is a variance parameter governing the magnitude of the exponential part of the kernel, $\ell$ is the $d$-dimensional vector of length scales (one for each input dimension), and the subscript $i$ refers to the $i^{\text {th }}$ component of the vector. These kernel parameters are referred to as hyperparameters in GPR. Thus, the hyperparameter vector for both the DPSE and the DPE kernels is $\left[\sigma_{0}^{2} \sigma_{1}^{2} \sigma_{2}^{2} \ldots \sigma_{d}^{2} \sigma_{f}^{2} \ell\right]^{T}$.

Numerous other kernel functions exist, details of which can be found in [29].

The noisy kernel hyperparameter vector $\theta$ is the kernel hyperparameter vector mentioned above with the noise variance $\sigma_{n}^{2}$ appended. It is estimated as the vector which maximizes the log posterior probability of the hyperparameter vector, given the matrix of input vectors $\mathrm{X}$ and the vector of dependent variable values y.

$$
\begin{aligned}
\hat{\theta}= & \underset{\theta}{\operatorname{argmax}} \log p(\theta \mid \mathbf{X}, \mathbf{y}) \\
= & \underset{\theta}{\operatorname{argmax}}\left\{\log p(\theta)-\frac{1}{2} \mathbf{y}^{\mathbf{T}} \mathbf{K}_{\mathbf{y}}^{-1} \mathbf{y}-\frac{1}{2} \log \left|\mathbf{K}_{\mathbf{y}}\right|\right. \\
& \left.-\frac{n}{2} \log (2 \pi)\right\}
\end{aligned}
$$

Here, $p(\theta)$ is the prior on the hyperparameter vector, $n$ is the number of observations, $\mathrm{X}$ is the $n \times d$ matrix of $d$-dimensional inputs, $\mathrm{y}$ is the $n \times 1$ vector of the dependent variable values, and $\mathrm{K}_{\mathrm{y}}$ is the $n \times n$ covariance matrix derived from the noisy kernel function over pairs of input variables (Eq. 14).

In this paper, the aim of GPR is to make predictions on new data points (and not hyperparameter inference, for example). For GPR, the predictive distribution of the dependent variable values $\mathrm{y}^{*}$ at a set of new inputs $\mathrm{X}^{*}$ is also a Gaussian distribution, given by

$$
\begin{aligned}
\mathbf{y}^{*} \mid \mathbf{X}^{*}, \mathcal{D}= & \mathcal{N}(\mu, C) \\
\mu= & \mathrm{K}\left(\mathrm{X}^{*}, \mathbf{X}\right) \mathbf{K}_{\mathbf{y}}^{-1} \mathbf{y} \\
C= & \mathbf{K}\left(\mathbf{X}^{*}, \mathbf{X}^{*}\right)-\mathbf{K}\left(\mathbf{X}^{*}, \mathbf{X}\right) \mathbf{K}_{\mathbf{y}}^{-1} \mathrm{~K}\left(\mathbf{X}^{*}, \mathbf{X}\right)^{\mathbf{T}} \\
& +\sigma_{n}^{2} \mathbf{I}_{\mathbf{n}^{*}} .
\end{aligned}
$$

Here, $n^{*}$ is the number of new inputs at which predictions are desired, $\mathrm{X}^{*}$ is the $n^{*} \times d$ matrix of the set of new inputs, $\mathcal{D}=(\mathrm{X}, \mathrm{y})$ is the set of training inputs and dependent variable values (used for hyperparameter inference), $\mathcal{N}(\mu, C)$ refers to a multivariate Gaussian distribution with mean vector $\mu$ and covariance matrix $C, \mathbf{K}\left(\mathbf{X}^{*}, \mathbf{X}\right)$ is the $n^{*} \times n$ covariance matrix derived from the noisy kernel function over pairs of new and training input variables (Eq. 14), $\mathrm{K}\left(\mathrm{X}^{*}, \mathrm{X}^{*}\right)$ is the $n^{*} \times n^{*}$ covariance matrix derived from the noisy kernel function over pairs of the new input variables, and $\mathbf{I}_{\mathbf{n}^{*}}$ is the $n^{*} \times n^{*}$ identity matrix.

Lastly, a few words about approximate GP inference are in order. The exact GP inference explained above involves inversion of an $n \times n$ matrix $\left(\mathrm{K}_{\mathrm{y}}\right)$, which requires $O\left(n^{3}\right)$ operations. As a result, exact GP inference can be computationally expensive for large data sets. In this paper, to reduce the computational burden, we use 
the Fully Independent Conditional (FIC) approximation to exact GP inference. Briefly, in this approximation, $m_{s}$ artificial input variables are introduced into the problem (called inducing inputs) with their associated dependent variable values (called inducing variables). The training function values and the function values at the new inputs (where predictions are desired) are all assumed fully independent among themselves, given the inducing variables. This approximation reduces the computational burden if $m_{s}$ is significantly less than $n$. More details of the FIC approximation can be found in [20].

GPR has several advantages. Firstly, it is a nonparametric method, thereby freeing the user from the choice of basis functions prior to model training (unlike, for example, least squares regression). Secondly, it retains a simple probabilistic framework based on the Gaussian distribution, making the analysis tractable (the tractability of the analysis is what makes the assumption of Gaussianity so useful in the first place). Thirdly, it is possible to determine the full predictive distribution, and therefore, prediction intervals accounting for the uncertainty in the output variable are a useful product of the analysis.

\section{MODEL DEVELOPMENT}

In this section, we explain the methodology employed to model the fuel flow rate using Gaussian Process Regression (GPR). Firstly, the A321-100 FDR data set is divided into training, validation, and test data sets. The training set comprises 76 flights (65\% of full data) randomly chosen from the full data set, the validation set comprises 18 flights (15\% of full data) randomly chosen from the full data set, and the test set comprises the remaining 23 flights (20\% of full data). The training set is used for model building, the validation set is used for model selection (from a pool of possible models), and the test set is used for evaluating the final selected model. The 76 flights in the training set result in a total of 18,261 observations in ascent, 933 observations in cruise, and 34,110 observations in descent.

All the variables are standardized, that is shifted by the sample mean and then scaled by the sample standard deviation of the respective variables in the training data sets. The GPR starts with hyperparameter inference for the different noisy kernel functions using the training data. The hyperparameters, being all positive, are given a broad gamma prior with mode 1 and variance 100 (for lack of specific prior knowledge). We use exact GP for inference in cruise (due to the small size of the cruise training data set). Due to the large size of the training data set in ascent and descent, we use GP with FIC approximation for inference in these phases. The FIC approximation is carried out by randomly pulling out 150 inducing variables from the training data set (the number of inducing variables is chosen by doing a selection study on the validation data set for the model predictive performance and the testing time). The freely available MATLAB ${ }^{\circledR}[1]$ based 'GPstuff Toolbox' [28] is used for GPR in this study. Once the models are trained and the hyperparameters are inferred, they are used to determine the model predictive distribution of the fuel flow rates for flights in the test data, as we will explain in the following subsection (Sec. 5.1).

\subsection{Prediction Algorithm}

The training data sets are used to train different models in ascent, cruise, and descent, using the variables described in Sec. 3. While training, the actual value of the instantaneous aircraft gross mass from the FDR data set is used as an input. However, the main aim of model building in our study is to use the models for predicting the fuel flow rate for new flights not used for training. For a new flight, the actual value of the instantaneous aircraft gross mass may not be available (since FDR data for the new flight could be proprietary and easily available ground-based tracking data cannot record the mass of the aircraft as it flies through the air). Hence, for a new flight, the value of the aircraft gross mass at a particular time instant needs to be estimated in order for it to be used as an input to further estimate the fuel flow rate at that time instant. In this section, we develop an algorithm which estimates both the instantaneous aircraft gross mass and the fuel flow rate for the airborne phases of a new flight. This algorithm operates on the knowledge that the reduction in gross mass with time is due to fuel burn only. For this algorithm to work, we assume that the values of the aircraft gross mass and the fuel flow rate are known at takeoff, which just precedes the first instant in ascent. These takeoff values, therefore, serve as initial conditions to the algorithm. At any instant of time, the aircraft gross mass is estimated using the estimated values of the gross mass and the fuel flow rate at the previous instant of time (Eq. 19). The estimated gross mass at the current instant then serves as one of the inputs (the exact values of the other inputs at that instant are assumed to be already available through ground-based tracking systems) to the fuel flow rate model trained for the particular phase (ascent/cruise/descent) in which the current time instant lies. This procedure continues till the end of descent.

$$
m_{i+1}=m_{i}-N_{\mathrm{eng}} \dot{m}_{f_{i}} \Delta t_{i+1 \leftarrow i}
$$

Here, $m_{i+1}$ and $m_{i}$ are the aircraft gross mass values at the $(i+1)^{\text {th }}$ and the $i^{\text {th }}$ time instants, respectively, $\dot{m}_{f_{i}}$ is the averaged fuel flow rate per engine at the $i^{\text {th }}$ time instant, and $\Delta t_{i+1 \leftarrow i}$ is the time interval between the $(i+1)^{\text {th }}$ and the $i^{\text {th }}$ time instants. The time interval between successive instants depends on the sampling frequency of the reported trajectory data for the new flight. The algorithm as such, does not need to assume an explicit value of the time step a priori.

In our algorithm, uncertain estimates of fuel flow rate are used to estimate the gross mass and these uncertain estimates of gross mass then serve as inputs to again estimate the fuel flow rate. It is important to correctly propagate these uncertainties from one time instant to the next. The regression problem in this case is hence, one having uncertainty in input values - a feature not encountered in common regression problems (in which the inputs are assumed to be free of any uncertainty). The uncertainty is in the gross mass input variable and not the other input variables whose exact values are assumed to be easily available. The approach described in [10] is suitably adopted to propagate the uncertainty in the input vector to the output. Let $\mathcal{D}$ be the training data set and $\mathrm{x}^{*}$ be a new uncertain input at which the prediction $y^{*}$ is desired. For correct propagation of uncertainty from input to output, we are interested in determining the probability of the output prediction given the training data, marginalized over all possible values of the uncertain 
input (Eq. 20). By using a Monte Carlo approach, the integral in Eq. 20 is approximated by an average (Eq. 21).

$$
\begin{aligned}
p\left(y^{*} \mid \mathcal{D}\right) & =\int p\left(y^{*} \mid \mathbf{x}^{*}, \mathcal{D}\right) p\left(\mathbf{x}^{*}\right) d \mathbf{x}^{*} \\
& \approx \frac{1}{n_{s}} \sum_{j=1}^{n_{s}} p\left(y^{*} \mid \mathbf{x}_{\mathbf{j}}^{*}, \mathcal{D}\right)
\end{aligned}
$$

where, $n_{s}$ is the number of samples and $\mathrm{x}_{\mathrm{j}}^{*}$ are samples of the uncertain input $\mathbf{x}^{*}$. For a GPR model, $p\left(y^{*} \mid \mathbf{x}_{\mathrm{j}}^{*}, \mathcal{D}\right)$ is a Gaussian density (Eq. 18). Thus, for a GPR model, $p\left(y^{*} \mid \mathcal{D}\right)$ can be approximated by a Gaussian Mixture density with $n_{s}$ number of components and equal component weights.

Algorithm 1 shows the algorithm for the fuel flow rate prediction. In the algorithm, subscripts 'prev' and 'curr' refer to the previous and the current time instants, respectively. 'IndexAscentStart' refers to the index of the point in the flight trajectory where ascent begins (the point immediately succeeding takeoff). 'IndexTouchdown' refers to the index of the point in the flight trajectory where the aircraft touches down at the arrival airport (just after the end of descent). $\mathbf{x}_{-\mathbf{m}}^{*}$ refers to the input vector where prediction is desired with the gross mass input variable removed. 'MeanPredGPR $\left(\mathbf{x}^{*}\right)$ ' and 'VarPredGPR $\left(\mathrm{x}^{*}\right)$ ' refer to the mean and the variance, respectively, of the prediction from the GPR fuel flow rate model (trained for the appropriate phase of flight, given in the subscripted parentheses) at a given input $x^{*}$. The subscript ' $1: n_{s}$ ' refers to the vector formed by taking all $n_{s}$ samples together. In this study, 100 samples are used for the Monte Carlo integration approach described in Eq 21 (the number of samples is chosen after considering the simulation time). $\mathcal{N}\left(\mu, \sigma^{2}\right)$ refers to a univariate Gaussian distribution with mean $\mu$ and variance $\sigma^{2} \cdot \mathcal{G M}\left(\mu, \sigma^{2}, \mathbf{w}\right)$ refers to a Gaussian Mixture distribution (of univariate Gaussians) with vector of component means $\mu$, vector of component variances $\sigma^{2}$, and vector of component weights $\mathbf{w}$. The mean of the vector of fuel flow rate samples $\left(\mathbf{m}_{\mathbf{f} \text { curr }}\right)$, generated according to Algorithm 1, gives the average predicted fuel flow rate per engine at a particular instant. The $95 \%$ confidence interval for the predicted fuel flow rate per engine at a particular instant is given by the inter-percentile range between the $2.5^{\text {th }}$ and the $97.5^{\text {th }}$ percentiles of the fuel flow rate samples $\left(\mathbf{m}_{\mathbf{f} \text { curr }}\right)$.

\section{MODEL EVALUATION}

The models are evaluated for their fuel flow rate predictive performance on flights in unseen data (not used for training). The mean model predictions and the $95 \%$ prediction intervals are calculated using the regression models developed in Sec. 5. The metrics used to evaluate the models are as follows:

- Mean Absolute Relative Prediction Error or Mean Error (ME): This is the mean of the absolute value of the relative prediction error on independent prediction data (validation or test data).

$$
\mathrm{ME}=\frac{1}{n^{*}} \sum_{i=1}^{n^{*}}\left|\frac{m_{f_{i}}-\hat{m}_{f_{i}}}{m_{f_{i}}}\right|
$$

Here, $n^{*}$ is the number of observations in the unseen prediction set, $\dot{m}_{f_{i}}$ is the actual fuel flow rate in the prediction set, and $\hat{m}_{f_{i}}$ is the model mean prediction of the fuel flow rate in the prediction set. The ME indicates the $L_{1}$-norm accuracy of the mean prediction.

- Normalized Root Mean Squared Prediction Error (NRMSPE): The NRMSPE indicates the $L_{2}$-norm accuracy of the mean prediction.

$$
\operatorname{NRMSPE}=\frac{\sqrt{\frac{1}{n^{*}} \sum_{i=1}^{n^{*}}\left(\dot{m}_{f_{i}}-\hat{\dot{m}}_{f_{i}}\right)^{2}}}{\operatorname{sd}\left(\hat{\mathbf{m}}_{\mathbf{f}}\right)}
$$

Here, $\operatorname{sd}\left(\hat{\mathbf{m}}_{\mathbf{f}}\right)$ is the standard deviation of the vector of the mean predicted fuel flow rates.

- Prediction Coverage (PC): This is the percentage of the observations in the prediction set for which the $95 \%$ prediction intervals given by the model include the actual values of the fuel flow rate. The PC gives an indication of the accuracy of the predictive uncertainty estimates.

Models having low ME, low NRMSPE, and high PC are preferred. The training phase results in GPR models built with different kernel functions. These metrics are used for model selection (using the unseen validation data set) and testing of the selected models (using the unseen test data set). The GPR model with the Dot Product Squared Exponential (DPSE) kernel is selected for ascent and descent, whereas the GPR model with the Dot Product Exponential (DPE) kernel is selected for cruise. Table 1 gives the predictive performance of the selected GPR models on unseen test data using the above metrics. It is seen that among the three phases, the performance of the GPR models is the poorest in descent. This poor performance could be a result of the propagation of uncertainty from takeoff (which serves as the initial condition to our algorithm) to descent (which is the farthest airborne phase from takeoff). It could also be a result of the high variability inherent in the descent phase due to operational reasons. Figure 2 shows the performance of the selected models on a particular flight in the test data set by plotting the model predictions against the actual fuel flow rate values and the time in flight.

\section{COMPARISON WITH BADA MODEL}

A commonly used model for estimating aircraft performance, including its fuel flow rate, is the Base of Aircraft Data (BADA) model developed by EUROCONTROL [15]. The BADA model is a total energy based model. It uses various simplistic empirical equations to estimate performance. These equations have aircraft type specific coefficients which are tabulated in a database. In this paper, we use the BADA Family 3 Thrust Specific Fuel Consumption (TSFC)-based fuel flow rate equations to estimate the BADA fuel flow rate. The net thrust required in these equations is derived from flight dynamics equations [9] applied to high fidelity FDR data. The thrust and the BADA fuel flow rate equations are described in Appendix B. Table 1 and Figure 2 show a comparison of our GPR model results with those given by the BADA model. Statistical tests are also used to compare the evaluation metrics for our GPR models and the BADA model. It is found that at a 5\% significance level, our models give statistically significantly better predictive performance than the BADA model for all the evaluation metrics in ascent, cruise, and descent (except for the Mean Error in cruise). In reality, the fuel 


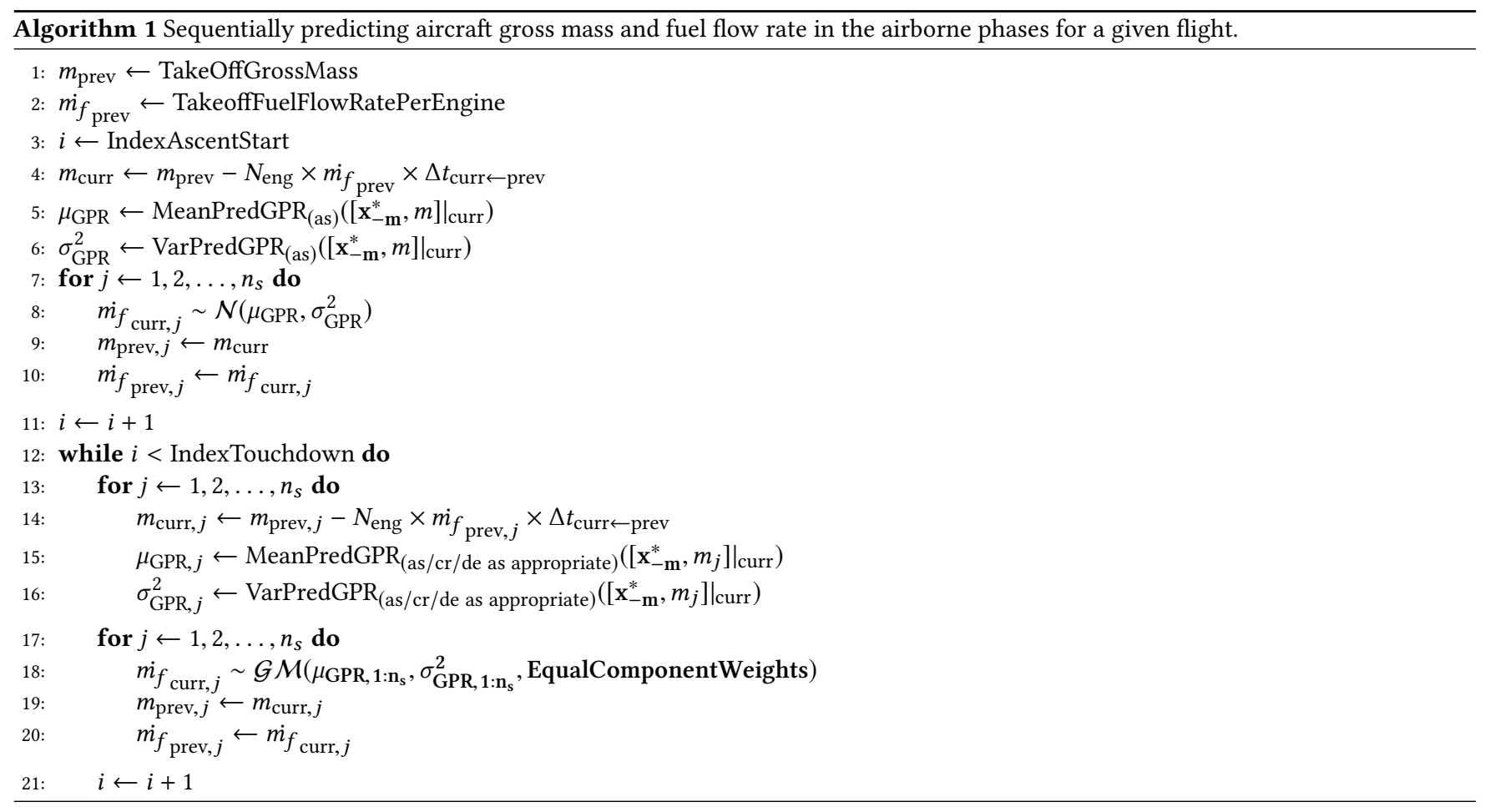

Table 1: Predictive performance of the GPR and the BADA models on A321-100 test data for the fuel flow rate. Each entry shows the mean and the standard deviation (within parentheses) of the evaluation metric across all the 23 flights in the test data. All the evaluation metrics are calculated on de-standardized data (that is, values at their original location and scale and not shifted by the mean and then scaled by the standard deviation of the training set variables).

\begin{tabular}{llll}
\hline \multirow{4}{*}{ Ascent } & $\begin{array}{l}\text { Model/ } \\
\text { Metric }\end{array}$ & GPR & BADA \\
& ME (\%) & $2.92(0.01)$ & $5.49(1.00)$ \\
& NRMSPE & $0.23(0.10)$ & $0.48(0.17)$ \\
& PC (\%) & $94.50(4.97)$ & $0(0)$ \\
\hline \multirow{3}{*}{ Cruise } & ME (\%) & $6.21(3.55)$ & $8.20(5.17)$ \\
& NRMSPE & $0.62(0.86)$ & $0.68(0.39)$ \\
& PC (\%) & $94.66(10.01)$ & $0(0)$ \\
\hline \multirow{3}{*}{ Descent } & ME (\%) & $15.34(2.85)$ & $23.56(4.62)$ \\
& NRMSPE & $0.49(0.11)$ & $0.84(0.34)$ \\
& PC (\%) & $92.10(6.49)$ & $0(0)$ \\
\hline
\end{tabular}

flow rate estimates from BADA are expected to be even worse than those in this study due to the use of low fidelity thrust values in practice (instead of the high fidelity FDR-based thrust values used here). Thus, the BADA results in this paper are quite optimistic. More importantly, being a deterministic model, BADA does not assign uncertainties to the fuel flow rate predictions (no prediction intervals), thereby giving a prediction coverage of zero. By contrast, our statistical GPR models are able to assign uncertainties to the fuel flow rate predictions in all the three phases (by giving prediction intervals), which enables one to quantify the variability seen in fuel flow rates due to operational reasons and unmodeled factors.

\section{CONCLUSIONS}

In this paper, an aircraft engine was considered as an example of a cyber-physical system whose performance is to be statistically analyzed through the fuel flow rate. Gaussian Process Regression (GPR) was used to statistically model the fuel flow rate.

Data from Flight Data Recorders (FDR) were used to capture the performance of a real engine in flight. Since fuel flow rate is an important variable governing engine performance, and flight data giving fuel flow rate sensor readings are proprietary to airlines, our models could be useful to researchers with limited access to flight data. They could also be used in the development of fuel burn and emissions inventories. With these aims in mind, we restricted our choice of variables to those available from flight track data. The instantaneous aircraft gross mass is an important predictor of fuel flow rate, but is not easily obtainable for a particular flight. We therefore, developed an algorithm which sequentially estimates the instantaneous gross mass and the fuel flow rate together, during the airborne phases of flight. The resulting GPR models were found to give mean errors of $2.9 \%, 6.2 \%$, and $15.3 \%$ in ascent, cruise, and descent, respectively. They were also found to perform statistically significantly (at a 5\% significance level) better than the Base of Aircraft Data (BADA) model which is currently used in practice, with up to a $50 \%$ reduction in mean error. Unlike the deterministic BADA model, our statistical GPR models were able to generate 

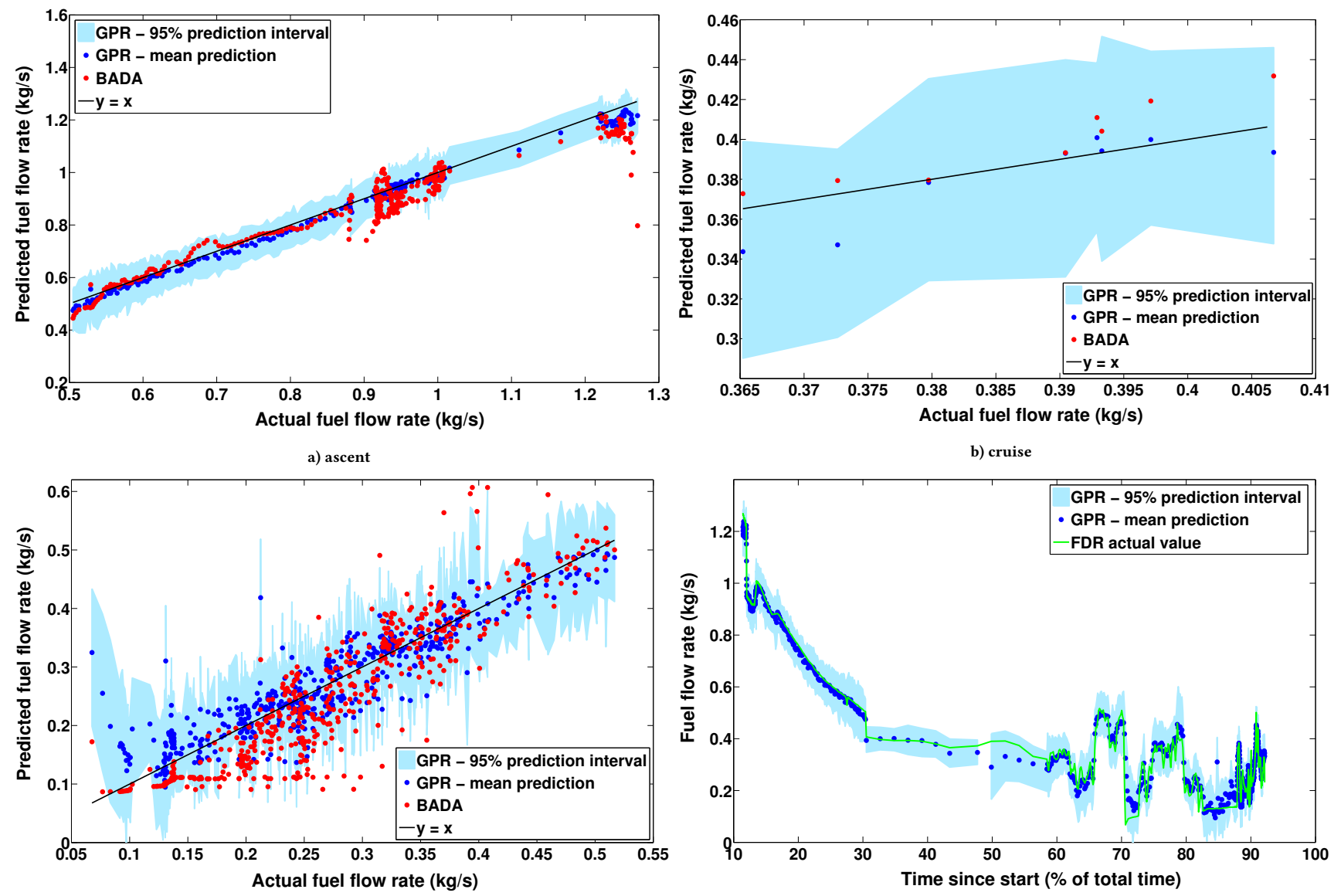

c) descent

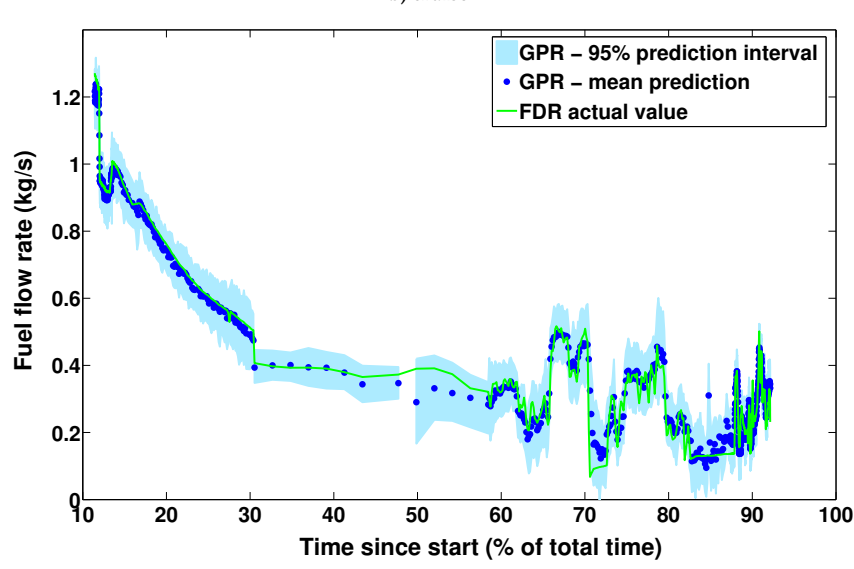

d) full airborne profile

Figure 2: A321-100 fuel flow rate: predictive performance of the GPR and the BADA models for one test data flight, in (a) ascent, (b) cruise, and (c) descent. The dots represent the mean predictions and the colored patch represents the $95 \%$ prediction intervals. The black line is the $y=x$ line for ease of comparison of the predicted and the actual fuel flow rates. (d) The predicted and the actual fuel flow rates in all the airborne phases as a function of time for the same one flight. The plots show de-standardized predicted and actual fuel flow rates.

interval estimates of the fuel flow rate predictions. These interval estimates reflect the cumulative variability in fuel flow rate across different flights due to operational reasons, meteorological conditions, random internal and external disturbances, and other unmodeled factors. Our GPR models generated prediction intervals with a prediction coverage of over $90 \%$ in all the airborne phases of flight.

The regression methodology proposed in this paper can be easily generalized to other applications where a statistical analysis of system performance using limited operational data is desired, especially where some of the input variables are uncertain (the aircraft mass in our specific application) and may need to be estimated in tandem with the output variable (the fuel flow rate in our case). Our novel algorithm automatically gives uncertainty estimates of the output variable and shows how uncertainties can be propagated over time. Moreover, our statistical method does not do away with domain expertise completely but continues to use a physical understanding of system dynamics for feature selection.

The results presented in this paper motivate promising directions for future research. We plan to analyze the sensitivity of our models to the simplifying assumptions adopted. Our algorithm assumes a knowledge of the takeoff mass, which may not be available for an individual flight. Therefore, we intend to develop methods to accurately estimate the takeoff mass. We also intend to use our algorithm to carry out similar fuel flow rate estimation for other aircraft types. The proposed algorithm in its current form is computationally intensive, as it carries out one-step predictions in a streaming fashion. In addition to the development of approaches better-suited to streaming data [4], future work will investigate methods for batch-processing points in the prediction set. 


\section{ACKNOWLEDGMENTS}

This work was supported in part by the US National Science Foundation under NSF Cyber-Physical Systems project FORCES, grant number 1239054. The views and conclusions contained in this document are those of the authors and should not be interpreted as representing the official policies, either expressed or implied, of the National Science Foundation.

\section{REFERENCES}

[1] 2012. The Mathworks, Inc., MATLAB ${ }^{\circledR}$ R2012a Documentation. (2012). Software Package.

[2] John David Anderson, Jr. 2004. Introduction to Flight (5 ${ }^{\text {th }}$ international ed.). McGraw-Hill Higher Education.

[3] C. A. L. Bailer-Jones, T. J. Sabin, D. J. C. MacKay, and P. J. Withers. 1997. Prediction of Deformed and Annealed Microstructures Using Bayesian Neural Networks and Gaussian Processes. In Proceedings of the Australasia-Pacific Forum on Intelligent Processing and Manufacturing of Materials.

[4] Shaunak D. Bopardikar and George S. Eskander Ekladious. 2016. Sequential Randomized Matrix Factorization for Gaussian Processes. In IEEE International Conference on Big Data (Big Data).

[5] Kian Ming A. Chai, Christopher K. I. Williams, Stefan Klanke, and Sethu Vijayakumar. 2009. Multi-Task Gaussian Process Learning of Robot Inverse Dynamics. In Advances in Neural Information Processing Systems 21. 265-272.

[6] Yashovardhan S. Chati and Hamsa Balakrishnan. 2014. Analysis of Aircraft Fuel Burn and Emissions in the Landing and Take Off Cycle using Operational Data In International Conference on Research in Air Transportation.

[7] Lei Clifton, David A. Clifton, Marco A. F. Pimentel, Peter J. Watkinson, and Lionel Tarassenko. 2012. Gaussian Process Regression in Vital-Sign Early Warning Systems. In Annual International Conference of the IEEE EMBS.

[8] Robert Dürichen, Marco A.F. Pimentel, Lei Clifton, Achim Schweikard, and David A. Clifton. 2014. Multi-Task Gaussian Process Models for Biomedical Applications. In IEEE-EMBS International Conference on Biomedical and Health Informatics.

[9] Bernard Etkin and Lloyd Duff Reid. 1996. Dynamics of Flight: Stability and Control $\left(3^{\text {rd }}\right.$ ed.). John Wiley \& Sons, Inc.

[10] Agathe Girard, Carl Edward Rasmussen, Joaquin Quiñonero-Candela, and Roderick Murray-Smith. 2003. Gaussian Process Priors With Uncertain Inputs Application to Multiple-Step Ahead Time Series Forecasting. In Advances in Neural Information Processing Systems 15. 545-552.

[11] Carolynne Hultquist, Gang Chen, and Kaiguang Zhao. 2014. A Comparison of Gaussian Process Regression, Random Forests, and Support Vector Regression for Burn Severity Assessment in Diseased Forests. Remote Sensing Letters 5, 8 (2014), 723-732.

[12] International Civil Aviation Organization. 2014. ICAO Aircraft Engine Emissions Databank. https://easa.europa.eu/document-library/ icao-aircraft-engine-emissions-databank. (2014). [Online; accessed 12 February 2014].

[13] Harshad Khadilkar and Hamsa Balakrishnan. 2012. Estimation of Aircraft Taxi Fuel Burn Using Flight Data Recorder Archives. Transportation Research Part D: Transport and Environment 17, 7 (2012), 532-537.

[14] Gennady G. Kulikov and Haydn A. Thompson (Eds.). 2004. Dynamic Modelling of Gas Turbines: Identification, Simulation, Condition Monitoring, and Optimal Control. Springer-Verlag, London.

[15] A. Nuic. 2015. User Manual for the Base of Aircraft Data (BADA) Revision 3.13 EUROCONTROL Experimental Centre. EEC Technical/Scientific Report No. 15/04/02-43.

[16] Luca Pasolli, Farid Melgani, and Enrico Blanzieri. 2010. Gaussian Process Regression for Estimating Chlorophyll Concentration in Subsurface Waters from Remote Sensing Data. IEEE Geoscience and Remote Sensing Letters 7, 3 (July 2010), 464-468.

[17] Judith Patterson, George J. Noel, David A. Senzig, Christopher J. Roof, and Gregg G. Fleming. 2009. Analysis of Departure and Arrival Profiles Using RealTime Aircraft Data. AIAA fournal of Aircraft 46, 4 (July-August 2009), 1094-1103.

[18] Marco A. F. Pimentel, David A. Clifton, Lei Clifton, and Lionel Tarassenko. 2013 Probabilistic Estimation of Respiratory Rate using Gaussian Processes. In Annual International Conference of the IEEE EMBS.
[19] John C. Platt, Christopher J. C. Burges, Steven Swenson, Christopher Weare, and Alice Zheng. 2002. Learning a Gaussian Process Prior for Automatically Generating Music Playlists. In Advances in Neural Information Processing Systems 14. $1425-1432$.

[20] Joaquin Quiñonero-Candela and Carl Edward Rasmussen. 2005. A Unifying View of Sparse Approximate Gaussian Process Regression. Journal of Machine Learning Research 6 (2005), 1939-1959.

[21] Carl Edward Rasmussen and Christopher K. I. Williams. 2006. Gaussian Processes for Machine Learning. The MIT Press.

[22] Megan S. Ryerson, Mark Hansen, and James Bonn. 2012. Validating Aircraft Performance Models with Airline Data. In International Conference on Research in Air Transportation.

[23] Anton Schwaighofer, Marian Grigoras, Volker Tresp, and Clemens Hoffmann. 2004. GPPS: A Gaussian Process Positioning System for Cellular Networks. In Advances in Neural Information Processing Systems 16. 579-586.

[24] David A. Senzig, Gregg G. Fleming, and Ralph J. Iovinelli. 2009. Modeling of Terminal-Area Airplane Fuel Consumption. AIAA fournal of Aircraft 46, 4 (July-August 2009), 1089-1093.

[25] Ashok N. Srivastava. 2012. Greener Aviation with Virtual Sensors: A Case Study. Data Mining and Knowledge Discovery 24, 2 (2012), 443-471.

[26] Alan J. Stolzer. 2003. Fuel Consumption Modeling of a Transport Category Aircraft: A Flight Operations Quality Assurance (FOQA) Analysis. Fournal of Air Transportation 8, 2 (2003), 3-18.

[27] A. A. Trani, F. C. Wing-Ho, G. Schilling, H. Baik, and A. Seshadri. 2004. A Neural Network Model to Estimate Aircraft Fuel Consumption. In AIAA Aviation Technology, Integration and Operations (ATIO) Forum.

[28] Jarno Vanhatalo, Jaakko Riihimäki, Jouni Hartikainen, Pasi Jylänki, Ville Tolvanen, and Aki Vehtari. 2013. GPstuff: Bayesian Modeling with Gaussian Processes. fournal of Machine Learning Research 14 (2013), 1175-1179.

[29] Jarno Vanhatalo, Jaakko Riihimäki, Jouni Hartikainen, Pasi Jylänki, Ville Tolvanen, and Aki Vehtari. 2015. Bayesian Modeling with Gaussian Processes using the GPstuff Toolbox. In arXiv:1206.5754 [stat.ML]

[30] Tim Woodbury and Ashok N. Srivastava. 2012. Analysis of Virtual Sensors for Predicting Aircraft Fuel Consumption. In AIAA Infotech@Aerospace.

[31] Tim Yoder. 2007. Development of Aircraft Fuel Burn Modeling Techniques with Applications to Global Emissions Modeling and Assessment of the Benefits of Reduced Vertical Separation Minimums. Master's thesis. Department of Aeronautics and Astronautics, Massachusetts Institute of Technology, Cambridge, MA, USA.

\section{APPENDICES}

\section{A INT'L STANDARD ATMOSPHERE}

According to the International Standard Atmosphere (ISA) model, the ambient air density $\rho$ is assumed to be a function of the altitude alone [2]

$$
\rho= \begin{cases}\rho_{\mathrm{SL}}\left(1+\beta \frac{h}{T_{\mathrm{SL}}}\right)^{-\frac{g}{\beta R}-1}, & \text { if } h<11000 \mathrm{~m}, \\ \rho_{11} \exp \left(\frac{-g}{R T_{11}}\left(h-h_{11}\right)\right), & \text { if } 11000 \mathrm{~m} \leq h<20000 \mathrm{~m}\end{cases}
$$

where, $\rho_{\mathrm{SL}}$ is the air density at sea level $\left(=1.225 \mathrm{kgm}^{-3}\right), \beta$ is the tropospheric lapse rate $\left(=-0.0065 \mathrm{Km}^{-1}\right), h$ is the altitude above mean sea level, $T_{\mathrm{SL}}$ is the air temperature at sea level $(=288.15 \mathrm{~K}), g$ is the acceleration due to gravity $\left(=9.81 \mathrm{~ms}^{-2}\right), R$ is the gas constant for air $\left(=287.05 \mathrm{Jkg}^{-1} \mathrm{~K}^{-1}\right), \rho_{11}$ is the air density $11000 \mathrm{~m}$ above sea level $\left(=0.364 \mathrm{kgm}^{-3}\right), T_{11}$ is the air temperature $11000 \mathrm{~m}$ above sea level $(=216.65 \mathrm{~K})$ and $h_{11}$ is the height of the tropopause start above mean sea level $(=11000 \mathrm{~m})$. The relative mean error between the ISA density and the actual density ranges from $0.30 \%-2.61 \%$ over the different A321-100 flights in our FDR data set. The low error indicates that the ISA densities are good approximations to the actual density values in the FDR. 


\section{B BADA FUEL FLOW RATE MODEL}

BADA uses the following equations for determining the averaged fuel flow rate per engine $\dot{m}_{f}$ [15]:

$$
\begin{aligned}
m_{f} & = \begin{cases}\frac{\mathrm{TSFC} \times F_{n}}{N_{\text {eng }}}, & \text { in ascent, } \\
\frac{\operatorname{TSFC} \times F_{n} \times C_{f \mathrm{cr}}}{N_{\text {eng }}}, & \text { in cruise, } \\
\frac{1}{N_{\text {eng }}} \max \left(\mathrm{TSFC} \times F_{n}, m_{f_{\text {min }}}\right), & \text { in descent }\end{cases} \\
\mathrm{TSFC} & =C_{f 1}\left(1+\frac{V}{C_{f 2}}\right) \\
m_{f_{\text {min }}}=C_{f 3}\left(1-\frac{h}{C_{f 4}}\right) &
\end{aligned}
$$

Here, TSFC is the Thrust Specific Fuel Consumption, $F_{n}$ is the aircraft net thrust from all engines, $N_{\text {eng }}$ is the number of engines, $V$ is the aircraft true air speed, $m_{f \text { min }}$ is the minimum aircraft fuel flow rate from all engines, and $h$ is the aircraft pressure altitude. $C_{f 1}, C_{f 2}, C_{f 3}, C_{f 4}$, and $C_{f \text { cr }}$ are aircraft type specific constants found in the BADA database. High fidelity FDR values are used for the true air speed, pressure altitude, and to calculate the net thrust.

The net thrust is calculated from FDR parameters using the following set of equations [9]:

$$
\begin{aligned}
F_{n}= & q \mathcal{S}\left(C_{D_{0}}+C_{D_{2}} C_{L}^{2}\right) \cos \alpha+m g \sin \theta_{\text {pitch }} \\
& -n_{w} m g \sin \alpha \cos \theta_{\text {bank }} \\
& +m\left(\dot{u}_{E}+q_{\mathrm{pitch}} V \sin \alpha+r c_{\text {wind }}\right) \\
q= & \frac{1}{2} \rho V^{2} \\
C_{L}= & \frac{n_{w} m g}{q \mathcal{S}} \\
\alpha= & \theta_{\text {pitch }}-\gamma \\
\sin \gamma= & \frac{\dot{h}}{V} \\
\sin \theta_{\text {bank }}= & \frac{1}{n_{w} g}\left(\dot{v}_{E}+r V \cos \alpha-r h_{\text {wind }}-p V \sin \alpha-\right. \\
& \left.g \cos \theta_{\text {pitch }} \sin \theta_{\text {roll }}\right) \\
\dot{u}_{E}= & \cos \alpha \frac{d V}{d t}-V \sin \alpha \frac{d \alpha}{d t}-\frac{d h_{\text {wind }}}{d t} \\
\dot{v}_{E}= & -\frac{d c_{\text {wind }}}{d t}(\text { assuming no sideslip })
\end{aligned}
$$

Here, $C_{L}$ is the aircraft lift coefficient, $\alpha$ is the angle of attack, $\theta_{\text {pitch }}$ is the pitch attitude, $n_{w}$ is the aircraft structural load factor (ratio of lift to weight), $\theta_{\mathrm{bank}}$ is the bank angle, $\dot{u}_{E}$ is the time derivative of the aircraft velocity along the roll axis in an inertial (ground fixed) frame, $q_{\text {pitch }}$ is the pitch rate, $r$ is the yaw rate, $c_{\text {wind }}$ is the crosswind (positive from starboard to port side), $h_{\text {wind }}$ is the headwind, $\dot{v}_{E}$ is the time derivative of the aircraft velocity along the pitch axis in an inertial (ground fixed) frame, $p$ is the roll rate, and $\theta_{\text {roll }}$ is the roll attitude.

The drag coefficients, $C_{D_{0}}$ and $C_{D_{2}}$ are calculated using the BADA equations [15] as follows:

$$
\begin{gathered}
C_{D_{0}}= \begin{cases}C_{D_{0, \mathrm{AP}}}, & \text { in approach phase, } \\
C_{D_{0, \mathrm{LDG}}}+C_{D_{0, \mathrm{ADG}}}, & \text { in landing phase, } \\
C_{D_{0, \mathrm{CR}}}, & \text { otherwise }\end{cases} \\
C_{D_{2}}= \begin{cases}C_{D_{2, \mathrm{AP}},}, & \text { in approach phase, } \\
C_{D_{2, \mathrm{LDG}},}, & \text { in landing phase, } \\
C_{D_{2, \mathrm{CR}}}, & \text { otherwise }\end{cases}
\end{gathered}
$$

The different coefficients in the right hand side of Eqs. $27-28$ are enumerated in the BADA database. In BADA, the approach phase is defined as either the part of descent where the pressure altitude above the arrival airport elevation is between the approach threshold altitude $\left(H_{\max , \mathrm{AP}}\right)$ and the landing threshold altitude $\left(H_{\text {max }, \mathrm{LD}}\right)$ with the calibrated air speed less than the minimum cruise speed increased by 10 knots, or the part of descent where the pressure altitude above the arrival airport elevation is less than the landing threshold altitude $\left(H_{\max , \mathrm{LD}}\right)$ with the calibrated air speed between 10 knots more than the minimum cruise speed and 10 knots more than the minimum approach speed. The landing phase is defined as the part of descent where the pressure altitude above the arrival airport elevation is less than the landing threshold altitude $\left(H_{\max , \mathrm{LD}}\right)$ with the calibrated air speed less than 10 knots more than the minimum approach speed. The values of the pressure altitude above the arrival airport elevation and the calibrated air speed are obtained from the FDR data set. The approach and landing threshold altitudes are obtained from the BADA database. The minimum speeds are calculated as follows:

$$
\begin{aligned}
V_{\text {min, cruise }} & =1.3 V_{\text {stall, cruise, ref }} \sqrt{\frac{m}{m_{\text {ref }}}} \\
V_{\text {min, approach }} & =1.3 V_{\text {stall, approach, ref }} \sqrt{\frac{m}{m_{\text {ref }}}}
\end{aligned}
$$

Here, $V_{\text {min, cruise }}$ and $V_{\text {min, approach }}$ are the minimum cruise and approach speeds, respectively. $m$ is the aircraft gross mass whose value is found in the FDR data set. $V_{\text {stall, cruise, ref }}$ and $V_{\text {stall, approach, ref }}$ are the reference stall speeds in cruise and approach, respectively whose values are found in the BADA database. All speeds are calibrated air speeds. $m_{\text {ref }}$ is the reference gross mass for the aircraft type and its value is also found in the BADA database. 